\title{
Some Aggregation Operators with Intuitionistic Trapezoid Fuzzy Linguistic Information and their Applications to Multi-Attribute Group Decision Making
}

\author{
Yanbing $\mathrm{Ju}^{1, *}$, Shanghong Yang ${ }^{1}$ and Aihua Wang ${ }^{2}$ \\ ${ }^{1}$ School of Management and Economics, Beijing Institute of Technology, Beijing 100081, China \\ ${ }^{2}$ Graduate School of Education, Peking University, Beijing 100871, China
}

Received: 10 Sep. 2013, Revised: 8 Dec. 2013, Accepted: 9 Dec. 2013

Published online: 1 Sep. 2014

\begin{abstract}
We study the multi-attribute group decision making (MAGDM) problems in which the attribute values provided by the decision makers take the form of intuitionistic trapezoid fuzzy linguistic (ITrFL) information considering the uncertainty and inaccuracy of input arguments. Some new aggregation operators called intuitionistic trapezoid fuzzy linguistic weighted average (ITrFLWA), intuitionistic trapezoid fuzzy linguistic ordered weighted average (ITrFLOWA) and intuitionistic trapezoid fuzzy linguistic hybrid weighted average (ITrFLHWA) operators are proposed at first. Then, we study some desirable properties of the proposed operators, such as monotonicity, idempotency, commutativity and boundedness. Next, two novel approaches based on the proposed operators are developed to solve MAGDM problems with intuitionistic trapezoid fuzzy linguistic information. Finally, an illustrative example of emergency logistics supplier selection is provided to verify the feasibility of the proposed approaches.
\end{abstract}

Keywords: Multi-attribute group decision making, Intuitionistic trapezoid fuzzy linguistic (ITrFL) information, Intuitionistic trapezoid fuzzy linguistic aggregation operators

\section{Introduction}

Multi-attribute group decision making (MAGDM) problem is one of the most important parts of decision theory. Due to the increasing complexity of the socioeconomic environment and the lack of knowledge or data about the group decision making problems domain, the attributes involved in the decision problems are not always expressed as crisp numbers, and some of them are more suitable to be denoted by fuzzy numbers, such as interval number, linguistic variable, intuitionistic fuzzy number etc. The fuzzy set theory (FS), initially introduced by Zadeh [1], is a good method to research MAGDM problems. Since Zadeh introduced fuzzy set theory to deal with vague problems, another useful tool called linguistic variables [2] are utilized to express a decision maker's preference information over objects in process of decision making under uncertain or vague environments. It makes evaluation by means of linguistic terms which describes qualitative linguistic information from 'extremely poor' to 'extremely good' and have been proven more practically and flexibly than FS. So far, a number of MAGDM approaches have been proposed for dealing with linguistic assessment information. Herrera et al. [3] presented a consensus model in group decision making under linguistic assessments. Herrera and Martłnez [4] developed 2-tuple linguistic approach which composed a linguistic term and a real number. Lin et al. [5] proposed the definition of interval-valued 2-tuple linguistic approach. Zhang [6, 7] presented some interval-valued 2-tuple linguistic aggregation operators. $\mathrm{Xu}$ [8] proposed uncertain linguistic variables which are the extension of linguistic variables. Recently, a new method called trapezoid fuzzy linguistic variables received lots of attention from researchers since it had been proposed by $\mathrm{Xu}$ [9], which is the generalized form of uncertain linguistic variables in essence but more suitable for processing vague information.

However, the fuzzy set is used to character the fuzziness just by membership degree. On the basis of the fuzzy set theory, Atanassov [10, 11] proposed the intuitionistic fuzzy set characterized by a membership

\footnotetext{
*Corresponding author e-mail: juyb@ bit.edu.cn
} 
function and a non-membership function. Obviously, the intuitionistic fuzzy set can describe and character the fuzzy essence of the objective world more exquisitely, and it has received more and more attention since its appearance [12-17].

In the real world, decision makers usually cannot completely express their opinions by a linguistic variable from a predefined linguistic term set or an intuitionistic fuzzy number, individually. Sometimes, they can express the information by combining linguistic variables and intuitionistic fuzzy set. Based on intuitionistic fuzzy set and linguistic variables, Wang and $\mathrm{Li}$ [18] proposed the concept of intuitionistic linguistic set, it can overcome the defects for intuitionistic fuzzy set which can only roughly represent criteria's membership and nonmembership to a particular concept, such as "good" and "bad", etc., and for linguistic variables which usually implies that membership degree is 1 , and the non-membership degree and hesitation degree of decision makers cannot be expressed [19]. Liu and Jin [20] defined the intuitionistic uncertain linguistic variables based on uncertain linguistic variables and intuitionistic fuzzy sets. Furthermore, Liu [21] defined the interval intuitionistic uncertain linguistic variables based on uncertain linguistic variables and interval-valued intuitionistic fuzzy sets (IVIFS).

In order to process uncertain and inaccuracy information more efficiency and precisely, it is necessary to make a further study on the extended form of the intuitionistic uncertain linguistic variables by combining trapezoid fuzzy linguistic variables and intuitionistic fuzzy set. For example, we can evaluate the transportation risk of the emergency logistics supplier by the linguistic set $S=\left\{s_{0}\right.$ (extremely low); $s_{1}$ (very low); $s_{2}$ (low); $s_{3}$ (medium); $s_{4}$ (high); $s_{5}$ (very high); $s_{6}$ (extremely high) $\}$. Perhaps, we can use the trapezoid fuzzy linguistic $\left[s_{\alpha}, s_{\beta}, s_{\theta}, s_{\tau}\right](0 \leq \alpha \leq \beta \leq \theta \leq \tau \leq 6)$ to describe the evaluation result, but this is not accurate, because it merely provides a linguistic range. In this situation, we can use an intuitionistic trapezoid fuzzy linguistic (ITrFL) $\left\langle\left[s_{\alpha}, s_{\beta}, s_{\theta}, s_{\tau}\right],(u, v)\right\rangle$ to describe the transport risk by giving the membership degree $u$ and non-membership degree $v$ to $\left[s_{\alpha}, s_{\beta}, s_{\theta}, s_{\tau}\right]$. This is the motivation of our study. As a fact, ITrFL avoids the information distortion and losing in the decision making process, and overcomes the shortcomings of the intuitionistic linguistic variables and the intuitionistic uncertain linguistic variables.

In this paper, a novel concept called intuitionistic trapezoid fuzzy linguistic variable which combines the trapezoid fuzzy linguistic variables and intuitionistic fuzzy set is proposed. Then some new operators for aggregating intuitionistic trapezoid fuzzy linguistic information are proposed, such as intuitionistic trapezoid fuzzy linguistic weighted average (ITrFLWA), intuitionistic trapezoid fuzzy linguistic ordered weighted average (ITrFLOWA) and intuitionistic trapezoid fuzzy linguistic hybrid weighted average (ITrFLHWA) operators. Furthermore, two novel methods to solve the MAGDM problems in which the attribute weights take the form of real numbers, attribute values take the form of intuitionistic trapezoid fuzzy linguistic information are developed based on the proposed operators. Finally, a numerical example of emergency logistics supplier selection is given to illustrate the applications of the developed methods.

The rest of this paper is organized as follows. In Section 2, some basic definitions of trapezoid fuzzy linguistic variables and intuitionistic linguistic numbers are reviewed, and intuitionistic trapezoid fuzzy linguistic variables is defined as well as operations, comparison and distance formula. In Section 3, we propose some new operators for aggregating intuitionistic trapezoid fuzzy linguistic information and study several desirable properties of these operators. In Section 4, two novel approaches for MAGDM based on the intuitionistic trapezoid fuzzy linguistic information are developed. In Section 5, a numerical example of emergency logistics supplier selection is given to illustrate the applications of the developed methods. The paper is concluded in Section 6.

\section{Preliminary}

\subsection{Trapezoid fuzzy linguistic variables}

A linguistic set is defined as a finite and completely ordered discrete term set, $S=\left\{s_{0}, s_{1}, \ldots, s_{l-1}\right\}$, where $l$ is the odd value. For example, when $l=7$, the linguistic term set $S$ can be defined as follows: $S=\left\{s_{0}\right.$ (extremely low); $s_{1}$ (very low); $s_{2}$ (low); $s_{3}$ (medium); $s_{4}$ (high); $s_{5}$ (very high); $s_{6}$ (extremely high) $\}$

Definition 2.1. [9] Let $\bar{S}=\left\{s_{\theta} \mid s_{0} \leq s_{\theta} \leq s_{l-1}, \theta \in[0, l-\right.$ $1]\}$, which is the continuous form of linguistic set $S$. $s_{\alpha}, s_{\beta}, s_{\theta}, s_{\tau}$ are four linguistic terms in $\bar{S}$, and $s_{0} \leq s_{\alpha} \leq s_{\beta} \leq s_{\theta} \leq s_{\tau} \leq s_{l-1}$ if $0 \leq \bar{\alpha} \leq \beta \leq \theta \leq \tau \leq l-1$, then the trapezoid fuzzy linguistic variable is defined as $\widetilde{s}=\left[s_{\alpha}, s_{\beta}, s_{\theta}, s_{\tau}\right]$, and $\widetilde{S}$ denotes a set of trapezoid fuzzy linguistic variables.

In particular, if any two of $\alpha, \beta, \theta, \tau$ are equal, then $\widetilde{s}$ is reduced to a triangular fuzzy linguistic variable; if any three of $\alpha, \beta, \theta, \tau$ are equal, then $\widetilde{s}$ is reduced to an uncertain linguistic variable.

\subsection{Intuitionistic linguistic numbers}

Based on intuitionistic fuzzy set and linguistic term set, Wang and $\mathrm{Li}$ [18] presented the extension form of the linguistic set, i.e., intuitionistic linguistic set, which is shown as follows.

Definition 2.2. [18] An intuitionistic linguistic set $A$ in $X$ can be defined as

$$
A=\left\{\left\langle x\left[s_{\theta(x)},(u(x), v(x))\right]\right\rangle \mid x \in X\right\}
$$


where $s_{\theta(x)} \in S, u(x) \in[0,1], \quad v(x) \in[0,1]$, and $u(x)+v(x) \leq 1, \forall x \in X . s_{\theta(x)}$ is a linguistic term, $u(x)$ represents the membership degree of the element $x$ to linguistic term $s_{\theta(x)}$, while $v(x)$ represents the non-membership degree of the element $x$ to linguistic term $s_{\theta(x)}$. Let $\pi(x)=1-u(x)-v(x), \pi(x) \in[0,1]$, $\forall x \in X$, then $\pi(x)$ is called a hesitancy degree of $x$ to linguistic term $s_{\theta(x)}$.

\subsection{Intuitionistic trapezoid fuzzy linguistic numbers}

Definition 2.3. [22] An intuitionistic trapezoid fuzzy linguistic set $\widetilde{A}$ in $X$ can be defined as

$$
\widetilde{A}=\left\{\left\langle x\left[\left[s_{\alpha(x)}, s_{\beta(x)}, s_{\theta(x)}, s_{\tau(x)}\right],(u(x), v(x))\right]\right\rangle \mid x \in X\right\}
$$

where $s_{\alpha(x)}, s_{\beta(x)}, s_{\theta(x)}, s_{\tau(x)} \in \tilde{S}, \quad u(x) \in[0,1]$, $v(x) \in[0,1]$, and $u(x)+v(x) \leq 1, \quad \forall x \in X$. $\left[s_{\alpha(x)}, s_{\beta(x)}, s_{\theta(x)}, s_{\tau(x)}\right]$ is a trapezoid fuzzy linguistic term, $u(x)$ represents the membership degree of the element $x$ to linguistic term $\left[s_{\alpha(x)}, s_{\beta(x)}, s_{\theta(x)}, s_{\tau(x)}\right]$, while $v(x)$ represents the non-membership degree of the element $x$ to linguistic term $\left[s_{\alpha(x)}, s_{\beta(x)}, s_{\theta(x)}, s_{\tau(x)}\right]$. Let $\pi(x)=1-u(x)-v(x), \pi(x) \in[0,1], \forall x \in X$, then $\pi(x)$ is called a hesitancy degree of $x$ to linguistic term $\left[s_{\alpha(x)}, s_{\beta(x)}, s_{\theta(x)}, s_{\tau(x)}\right]$.

In Eq. (2) $\left\langle\left[s_{\alpha(x)}, s_{\beta(x)}, s_{\theta(x)}, s_{\tau(x)}\right],(u(x), v(x))\right\rangle$ is an intuitionistic trapezoid fuzzy linguistic number (ITrFLN). Obviously, $\widetilde{A}$ can be viewed as a collection of intuitionistic trapezoid fuzzy linguistic numbers (ITrFLNs). For convenience, $\widetilde{a}=\left\langle\left[s_{\alpha(\widetilde{a})}, s_{\beta(\widetilde{a})}, s_{\theta(\widetilde{a})}, s_{\tau(\widetilde{a})}\right],(u(\widetilde{a}), v(\widetilde{a}))\right\rangle$ is used to represent an ITrFLN.

$\begin{array}{llll}\text { Definition } & \text { 2.4. } & \text { Let }\end{array}$ $\widetilde{a}_{i}=\left\langle\left[s_{\alpha\left(\widetilde{a}_{i}\right)}, s_{\beta\left(\widetilde{a}_{i}\right)}, s_{\theta\left(\widetilde{a}_{i}\right)}, s_{\tau\left(\widetilde{a}_{i}\right)}\right],\left(u\left(\widetilde{a}_{i}\right), v\left(\widetilde{a}_{i}\right)\right)\right\rangle \quad$ and $\widetilde{a}_{j}=\left\langle\left[s_{\alpha\left(\widetilde{a}_{j}\right)}, s_{\beta\left(\widetilde{a}_{j}\right)}, s_{\theta\left(\widetilde{a}_{j}\right)}, s_{\tau\left(\widetilde{a}_{j}\right)}\right],\left(u\left(\widetilde{a}_{j}\right), v\left(\widetilde{a}_{j}\right)\right)\right\rangle$ be two ITrFLNs and $\lambda \geq 0$, then the operations of ITrFLNs can be defined as Eqs. (3-6).

\section{Theorem}

2.1.

Let $\widetilde{a}_{i}=\left\langle\left[s_{\alpha\left(\widetilde{a}_{i}\right)}, s_{\beta\left(\widetilde{a}_{i}\right)}, s_{\theta\left(\widetilde{a}_{i}\right)}, s_{\tau\left(\widetilde{a}_{i}\right)}\right],\left(u\left(\widetilde{a}_{i}\right), v\left(\widetilde{a}_{i}\right)\right)\right\rangle \quad$ and $\widetilde{a}_{j}=\left\langle\left[s_{\alpha\left(\widetilde{a}_{j}\right)}, s_{\beta\left(\widetilde{a}_{j}\right)}, s_{\theta\left(\widetilde{a}_{j}\right)}, s_{\tau\left(\widetilde{a}_{j}\right)}\right],\left(u\left(\widetilde{a}_{j}\right), v\left(\widetilde{a}_{j}\right)\right)\right\rangle$ be two ITrFLNs and $\lambda, \lambda_{i}, \lambda_{j} \geq 0$, then we can obtain the following rules.

$$
\begin{aligned}
& \text { 1. } \widetilde{a}_{i} \oplus \widetilde{a}_{j}=\widetilde{a}_{j} \oplus \widetilde{a}_{i} \\
& \text { 2. } \widetilde{a}_{i} \otimes \widetilde{a}_{j}=\widetilde{a}_{j} \otimes \widetilde{a}_{i} \\
& \text { 3. } \lambda\left(\widetilde{a}_{i} \oplus \widetilde{a}_{j}\right)=\lambda \widetilde{a}_{i} \oplus \lambda \widetilde{a}_{j} \\
& \text { 4. } \lambda_{i} \widetilde{a}_{i} \oplus \lambda_{j} \widetilde{a}_{i}=\left(\lambda_{i}+\lambda_{j}\right) \widetilde{a}_{i} \\
& \text { 5. } \tilde{a}_{i}^{\lambda_{i}} \otimes \widetilde{a}_{i}^{\lambda_{j}}=\tilde{a}_{i}^{\lambda_{i}+\lambda_{j}} \\
& \text { 6. } \tilde{a}_{i}^{\lambda} \otimes \widetilde{a}_{j}^{\lambda}=\left(\widetilde{a}_{i} \otimes \widetilde{a}_{j}\right)^{\lambda_{j}}
\end{aligned}
$$

\section{Definition}

2.5.
ITrFLN, the expected function $E\left(\widetilde{a}_{i}\right)$ and the accuracy function $H\left(\widetilde{a}_{i}\right)$ of $\widetilde{a}_{i}$ are defined as Eqs. (7-8), respectively.

Assume that $\widetilde{a}_{i}$ and $\widetilde{a}_{j}$ are two ITrFLNs, they can be compared by the following rules:

$$
\begin{aligned}
& \text { 1.If } E\left(\widetilde{a}_{i}\right)>E\left(\widetilde{a}_{j}\right) \text {, then } \widetilde{a}_{i}>\widetilde{a}_{j} ; \\
& \text { 2.If } E\left(\widetilde{a}_{i}\right)=E\left(\widetilde{a}_{j}\right) \text {, then } \\
& \quad \text { if } H\left(\widetilde{a}_{i}\right)>H\left(\widetilde{a}_{j}\right) \text {, then } \widetilde{a}_{i}>\widetilde{a}_{j} ; \\
& \quad \text { if } H\left(\widetilde{a}_{i}\right)=H\left(\widetilde{a}_{j}\right) \text {, then } \widetilde{a}_{i}=\widetilde{a}_{j} ; \\
& \quad \text { if } H\left(\widetilde{a}_{i}\right)<H\left(\widetilde{a}_{j}\right) \text {, then } \widetilde{a}_{i}<\widetilde{a}_{j} ;
\end{aligned}
$$

\section{Definition \\ 2.6. $\widetilde{a}_{i}=\left\langle\left[s_{\alpha\left(\widetilde{a}_{i}\right)}, s_{\beta\left(\widetilde{a}_{i}\right)}, s_{\theta\left(\widetilde{a}_{i}\right)}, s_{\tau\left(\widetilde{a}_{i}\right)}\right],\left(u\left(\widetilde{a}_{i}\right), v\left(\widetilde{a}_{i}\right)\right)\right\rangle \quad$ and $\widetilde{a}_{j}=\left\langle\left[s_{\alpha\left(\widetilde{a}_{j}\right)}, s_{\beta\left(\widetilde{a}_{j}\right)}, s_{\theta\left(\widetilde{a}_{j}\right)}, s_{\tau\left(\widetilde{a}_{j}\right)}\right],\left(u\left(\widetilde{a}_{j}\right), v\left(\widetilde{a}_{j}\right)\right)\right\rangle$ be two ITrFLNs, the normalized Hamming distance between $\widetilde{a}_{i}$ and $\widetilde{a}_{j}$ is defined as Eq. (9). \\ 3 Some aggregation operators based on intuitionistic trapezoid fuzzy linguistic numbers}

Let

Based on the ordered weighted average (OWA) operator [23], we define three new operators such as intuitionistic trapezoid fuzzy linguistic weighted average (ITrFLWA) operator, intuitionistic trapezoid fuzzy linguistic ordered weighted average (ITrFLOWA) operator and intuitionistic trapezoid fuzzy linguistic hybrid weighted average (ITrFLHWA) operator in what follows.

\section{Definition}

3.1.

Let $\widetilde{a}_{j}=\left\langle\left[s_{\alpha\left(\widetilde{a}_{j}\right)}, s_{\beta\left(\widetilde{a}_{j}\right)}, s_{\theta\left(\widetilde{a}_{j}\right)}, s_{\tau\left(\widetilde{a}_{j}\right)}\right],\left(u\left(\widetilde{a}_{j}\right), v\left(\widetilde{a}_{j}\right)\right)\right\rangle$ $(j=1,2, \ldots, n)$ be a collection of the ITrFLNs. The intuitionistic trapezoid fuzzy linguistic weighted average (ITrFLWA) operator can be defined as follows, and ITrFLWA: $\Omega^{n} \rightarrow \Omega$ :

$$
\operatorname{ITrFLWA}\left(\widetilde{a}_{1}, \widetilde{a}_{2}, \ldots, \widetilde{a}_{n}\right)=\sum_{j=1}^{n} \omega_{j} \widetilde{a}_{j}
$$

where $\Omega$ is the set of all intuitionistic trapezoid fuzzy linguistic numbers, and $\omega=\left(\omega_{1}, \omega_{2}, \ldots, \omega_{n}\right)^{T}$ is the weight vector of $\widetilde{a}_{j}(j=1,2, \ldots, n)$, such that $\omega \in[0,1]$, $\sum_{j=1}^{n} \omega_{j}=1$.

Especially, if $\omega=\left(\frac{1}{n}, \frac{1}{n}, \ldots, \frac{1}{n}\right)^{T}$, then the ITrFLWA operator will be simplified to an intuitionistic trapezoid fuzzy linguistic average (ITrFLA) operator.

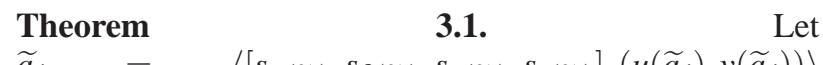
$\widetilde{a}_{j}=\left\langle\left[s_{\alpha\left(\widetilde{a}_{j}\right)}, s_{\beta\left(\widetilde{a}_{j}\right)}, s_{\theta\left(\widetilde{a}_{j}\right)}, s_{\tau\left(\widetilde{a}_{j}\right)}\right],\left(u\left(\widetilde{a}_{j}\right), v\left(\widetilde{a}_{j}\right)\right)\right\rangle$ $(j=1,2, \ldots, n)$ be a collection of the ITrFLNs. Eq. (10) can be transformed into Eq. (11), which is still an ITrFLN.

Theorem 3.1 can be proven by mathematical induction. The steps in the proof are as follows:

Proof.

1.When $n=1$, obviously, Eq. (11) is correct. 


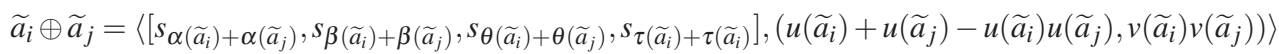

$$
\begin{aligned}
& \widetilde{a}_{i} \otimes \widetilde{a}_{j}=\left\langle\left[s_{\alpha\left(\widetilde{a}_{i}\right) \times \alpha\left(\widetilde{a}_{j}\right)}, s_{\beta\left(\widetilde{a}_{i}\right) \times \beta\left(\widetilde{a}_{j}\right)}, s_{\theta\left(\widetilde{a}_{i}\right) \times \theta\left(\widetilde{a}_{j}\right)}, s_{\tau\left(\widetilde{a}_{i}\right) \times \tau\left(\widetilde{a}_{i}\right)}\right],\left(u\left(\widetilde{a}_{i}\right) u\left(\widetilde{a}_{j}\right), v\left(\widetilde{a}_{i}\right)+v\left(\widetilde{a}_{j}\right)-v\left(\widetilde{a}_{i}\right) v\left(\widetilde{a}_{j}\right)\right)\right\rangle \\
& \lambda \widetilde{a}_{i}=\left\langle\left[s_{\lambda \times \alpha\left(\widetilde{a}_{i}\right)}, s_{\lambda \times \beta\left(\widetilde{a}_{i}\right)}, s_{\lambda \times \theta\left(\widetilde{a}_{i}\right)}, s_{\lambda \times \tau\left(\widetilde{a}_{i}\right)}\right],\left(1-\left(1-u\left(\widetilde{a}_{i}\right)^{\lambda}\right),\left(v\left(\widetilde{a}_{i}\right)^{\lambda}\right)\right\rangle\right.
\end{aligned}
$$

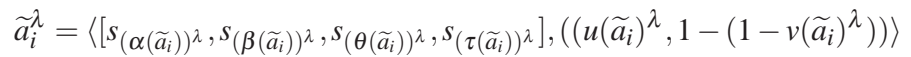

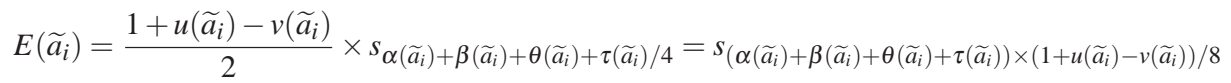

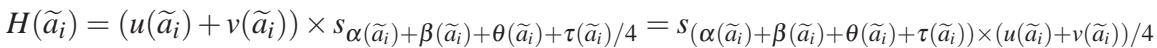

$\operatorname{ITrFLWA}\left(\widetilde{a}_{1}, \widetilde{a}_{2}, \ldots, \widetilde{a}_{n}\right)=\left\langle\left[s_{\sum_{j=1}^{n} \omega_{j} \times \alpha\left(\widetilde{a}_{j}\right)}, s_{\sum_{j=1}^{n} \omega_{j} \times \beta\left(\widetilde{a}_{j}\right)}, s_{\sum_{j=1}^{n} \omega_{j} \times \theta\left(\widetilde{a}_{j}\right)}, s_{\sum_{j=1}^{n} \omega_{j} \times \tau\left(\widetilde{a}_{j}\right)}\right],\left(1-\prod_{j=1}^{n}\left(1-u\left(\widetilde{a}_{j}\right)\right)\right)^{\omega_{j}}, \prod_{j=1}^{n}\left(v\left(\widetilde{a}_{j}\right)\right)^{\omega_{j}}\right\rangle$

2.When $n=2$, since

$$
\begin{aligned}
\omega_{1} \widetilde{a}_{1}= & \left\langle\left[s_{\omega_{1} \times \alpha\left(\widetilde{a}_{1}\right)}, s_{\omega_{1} \times \beta\left(\widetilde{a}_{1}\right)}, s_{\omega_{1} \times \theta\left(\widetilde{a}_{1}\right)}, s_{\omega_{1} \times \tau\left(\widetilde{a}_{1}\right)}\right],\right. \\
& \left.\left(1-\left(1-u\left(\widetilde{a}_{1}\right)\right)\right)^{\omega_{1}},\left(v\left(\widetilde{a}_{1}\right)\right)^{\omega_{1}}\right\rangle \\
\omega_{1} \widetilde{a}_{2}= & \left\langle\left[s_{\omega_{2} \times \alpha\left(\widetilde{a}_{2}\right)}, s_{\omega_{1} \times \beta\left(\widetilde{a}_{2}\right)}, s_{\omega_{1} \times \theta\left(\widetilde{a}_{2}\right)}, s_{\omega_{2} \times \tau\left(\widetilde{a}_{2}\right)}\right],\right. \\
& \left.\left(1-\left(1-u\left(\widetilde{a}_{2}\right)\right)\right)^{\omega_{2}},\left(v\left(\widetilde{a}_{2}\right)\right)^{\omega_{2}}\right\rangle
\end{aligned}
$$

thus,

$$
\begin{aligned}
& \operatorname{ITrFLWA}\left(\widetilde{a}_{1}, \widetilde{a}_{2}\right) \\
& =\omega_{1} \widetilde{a}_{1}+\omega_{2} \widetilde{a}_{2} \\
& =\left(\left\langle\left[s_{\omega_{1} \times \alpha\left(\widetilde{a}_{1}\right)}, s_{\omega_{1} \times \beta\left(\widetilde{a}_{1}\right)}, s_{\omega_{1} \times \theta\left(\widetilde{a}_{1}\right)} s_{\omega_{1} \times \tau\left(\widetilde{a}_{1}\right)}\right],\right.\right. \\
& \left.\left.\left(1-\left(1-u\left(\widetilde{a}_{1}\right)\right)\right)^{\omega_{1}},\left(v\left(\widetilde{a}_{1}\right)\right)^{\omega_{1}}\right\rangle\right) \\
& +\left(\left\langle\left[s_{\omega_{2} \times \alpha\left(\widetilde{a}_{2}\right)}, s_{\omega_{2} \times \beta\left(\widetilde{a}_{2}\right)}, s_{\omega_{2} \times \theta\left(\widetilde{a}_{2}\right)} s_{\omega_{2} \times \tau\left(\widetilde{a}_{2}\right)}\right]\right.\right. \text {, } \\
& \left.\left.\left(1-\left(1-u\left(\widetilde{a}_{2}\right)\right)\right)^{\omega_{2}},\left(v\left(\widetilde{a}_{2}\right)\right)^{\omega_{2}}\right\rangle\right) \\
& =\left\langle\left[ s_{\sum_{j=1}^{2} \omega_{j} \times \alpha\left(\widetilde{a}_{j}\right)}, s_{\sum_{j=1}^{2} \omega_{j} \times \beta\left(\widetilde{a}_{j}\right)},\right.\right. \\
& \left.s_{\sum_{j=1}^{2} \omega_{j} \times \theta\left(\widetilde{a}_{j}\right)}, s_{\sum_{j=1}^{2} \omega_{j} \times \tau\left(\widetilde{a}_{j}\right)}\right], \\
& \left.\left(1-\prod_{j=1}^{2}\left(1-u\left(\widetilde{a}_{j}\right)\right)\right)^{\omega_{j}}, \prod_{j=1}^{2}\left(v\left(\widetilde{a}_{j}\right)\right)^{\omega_{j}}\right\rangle
\end{aligned}
$$

3.Suppose that when $n=k$, Eq. (11) is correct, i.e.,

$$
\begin{aligned}
& \operatorname{ITrFLWA}\left(\widetilde{a}_{1}, \widetilde{a}_{2}, \ldots, \widetilde{a}_{k}\right) \\
= & \left\langle\left[ s_{\sum_{j=1}^{k} \omega_{j} \times \alpha\left(\widetilde{a}_{j}\right)}, s_{\sum_{j=1}^{k} \omega_{j} \times \beta\left(\widetilde{a}_{j}\right)},\right.\right. \\
& \left.s_{\sum_{j=1}^{k} \omega_{j} \times \theta\left(\widetilde{a}_{j}\right)}, s_{\sum_{j=1}^{k} \omega_{j} \times \tau\left(\widetilde{a}_{j}\right)}\right], \\
& \left.\left(1-\prod_{j=1}^{k}\left(1-u\left(\widetilde{a}_{j}\right)\right)\right)^{\omega_{j}}, \prod_{j=1}^{k}\left(v\left(\widetilde{a}_{j}\right)\right)^{\omega_{j}}\right\rangle
\end{aligned}
$$

then, when $n=k+1$, we have

$$
\begin{aligned}
& \operatorname{ITrFLWA}\left(\widetilde{a}_{1}, \widetilde{a}_{2}, \ldots, \widetilde{a}_{k}, \widetilde{a}_{k+1}\right) \\
= & \left\langle\left[ s_{\sum_{j=1}^{k} \omega_{j} \times \alpha\left(\widetilde{a}_{j}\right)}, s_{\sum_{j=1}^{k} \omega_{j} \times \beta\left(\widetilde{a}_{j}\right)},\right.\right. \\
& \left.s_{\sum_{j=1}^{k} \omega_{j} \times \theta\left(\widetilde{a}_{j}\right)}, s_{\sum_{j=1}^{k} \omega_{j} \times \tau\left(\widetilde{a}_{j}\right)}\right], \\
& \left.\left(1-\prod_{j=1}^{k}\left(1-u\left(\widetilde{a}_{j}\right)\right)\right)^{\omega_{j}}, \prod_{j=1}^{k}\left(v\left(\widetilde{a}_{j}\right)\right)^{\omega_{j}}\right\rangle \\
& +\left(\left\langle\left[s_{\omega_{k+1} \times \alpha\left(\widetilde{a}_{k+1}\right)}, s_{\omega_{k+1} \times \beta\left(\widetilde{a}_{k+1}\right)},\right.\right.\right. \\
& \left.s_{\omega_{k+1} \times \theta\left(\widetilde{a}_{k+1}\right)} s_{\omega_{k+1} \times \tau\left(\widetilde{a}_{k+1}\right)}\right], \\
& \left(1-\left(1-u\left(\widetilde{a}_{k+1}\right)\right)\right)^{\omega_{k+1}},\left(v\left(\widetilde{a}_{k+1}\right)\right)^{\left.\left.\omega_{k+1}\right\rangle\right)} \\
= & \left\langle\left[ s_{\sum_{j=1}^{k+1} \omega_{j} \times \alpha\left(\widetilde{a}_{j}\right)}, s_{\sum_{j=1}^{k+1} \omega_{j} \times \beta\left(\widetilde{a}_{j}\right)},\right.\right. \\
& \left.s_{\sum_{j=1}^{k+1} \omega_{j} \times \theta\left(\widetilde{a}_{j}\right)}, s_{\sum_{j=1}^{k+1} \omega_{j} \times \tau\left(\widetilde{a}_{j}\right)}\right], \\
& \left(1-\prod_{j=1}^{k+1}\left(1-u\left(\widetilde{a}_{j}\right)\right)\right)^{\omega_{j}}, \prod_{j=1}^{k+1}\left(v\left(\widetilde{a}_{j}\right)\right)^{\left.\omega_{j}\right\rangle}
\end{aligned}
$$

therefore, when $n=k+1$, Eq. (11) is correct as well. Thus, Eq. (11) is correct for all $n$.

Theorem 3.2. (Monotonicity) Let $\left(\widetilde{a}_{1}, \widetilde{a}_{2}, \ldots, \widetilde{a}_{n}\right)$ and $\left(\widetilde{a}_{1}^{\prime}, \widetilde{a}_{2}^{\prime}, \ldots, \widetilde{a}_{n}^{\prime}\right)$ be two collections of ITrFLNs. For all $j=1,2, \ldots, n$, if $\widetilde{a}_{j}^{\prime} \leq \widetilde{a}_{j}$, then

$\operatorname{ITrFLWA}\left(\widetilde{a}_{1}, \widetilde{a}_{2}, \ldots, \widetilde{a}_{n}\right) \leq \operatorname{ITrFLWA}\left(\widetilde{a}_{1}, \widetilde{a}_{2}, \ldots, \widetilde{a}_{n}\right)$ 
Proof. According to the definition of ITrFLWA in Eq. (11), we have

$$
\begin{aligned}
& \operatorname{ITrFLWA}\left(\widetilde{a}_{1}^{\prime}, \tilde{a}_{2}, \ldots, \widetilde{a}_{n}^{\prime}\right) \\
& =\left\langle\left[ s_{\sum_{j=1}^{n} \omega_{j} \times \alpha\left(\widetilde{a}_{j}\right)}, s_{\sum_{j=1}^{n} \omega_{j} \times \beta\left(\widetilde{a}_{j}\right)},\right.\right. \\
& \left.s_{\sum_{j=1}^{n} \omega_{j} \times \theta\left(\widetilde{a}_{j}^{\prime}\right)}, s_{\sum_{j=1}^{n} \omega_{j} \times \tau\left(\widetilde{a}_{j}^{\prime}\right)}\right), \\
& \left.\left(1-\prod_{j=1}^{n}\left(1-u\left(\tilde{a}_{j}^{\prime}\right)\right)\right)^{\omega_{j}}, \prod_{j=1}^{n}\left(v\left(\tilde{a}_{j}\right)\right)^{\omega_{j}}\right\rangle \\
& \operatorname{ITrFLWA}\left(\tilde{a}_{1}, \widetilde{a}_{2}, \ldots, \tilde{a}_{n}\right) \\
& =\left\langle\left[ s_{\sum_{j=1}^{n} \omega_{j} \times \alpha\left(\widetilde{a}_{j}\right)}, s_{\sum_{j=1}^{n} \omega_{j} \times \beta\left(\widetilde{a}_{j}\right)},\right.\right. \\
& \left.s_{\sum_{j=1}^{n} \omega_{j} \times \theta\left(\widetilde{a}_{j}\right)}, s_{\sum_{j=1}^{n} \omega_{j} \times \tau\left(\widetilde{a}_{j}\right)}\right], \\
& \left.\left(1-\prod_{j=1}^{n}\left(1-u\left(\widetilde{a}_{j}\right)\right)\right)^{\omega_{j}}, \prod_{j=1}^{n}\left(v\left(\widetilde{a}_{j}\right)\right)^{\omega_{j}}\right\rangle
\end{aligned}
$$

and their expected values can be calculated by Eq. (7)

$$
\begin{aligned}
& E\left(\operatorname{ITrFLWA}\left(\widetilde{a}_{1}, \widetilde{a}_{2}, \ldots, \widetilde{a}_{n}\right)\right)= \\
& s_{\left(\sum_{j=1}^{n} \omega_{j} \times \alpha\left(\widetilde{a}_{j}^{\prime}\right)+\sum_{j=1}^{n} \omega_{j} \times \beta\left(\widetilde{a}_{j}^{\prime}\right)+\sum_{j=1}^{n} \omega_{j} \times \theta\left(\widetilde{a}_{j}^{\prime}\right)+\sum_{j=1}^{n} \omega_{j} \times \tau\left(\widetilde{a}_{j}\right)\right)} \\
& \left.\times\left(1+\left(1-\prod_{j=1}^{n}\left(1-u\left(\vec{a}_{j}^{\prime}\right)\right)^{\omega_{j}}\right)-\prod_{j=1}^{n}\right)\left(v\left(\vec{a}_{j}\right)\right)^{\omega_{j}}\right) / 8 \\
& E\left(\operatorname{ITrFLWA}\left(\widetilde{a}_{1}, \widetilde{a}_{2}, \ldots, \widetilde{a}_{n}\right)\right)= \\
& s_{\left(\sum_{j=1}^{n} \omega_{j} \times \alpha\left(\widetilde{a}_{j}\right)+\sum_{j=1}^{n} \omega_{j} \times \beta\left(\widetilde{a}_{j}\right)+\sum_{j=1}^{n} \omega_{j} \times \theta\left(\widetilde{a}_{j}\right)+\sum_{j=1}^{n} \omega_{j} \times \tau\left(\widetilde{a}_{j}\right)\right)} \\
& \left.\times\left(1+\left(1-\prod_{j=1}^{n}\left(1-u\left(\widetilde{a}_{j}\right)\right)^{\omega_{j}}\right)-\prod_{j=1}^{n}\right)\left(v\left(\widetilde{a}_{j}\right)\right)^{\omega_{j}}\right) / 8
\end{aligned}
$$

since $\tilde{a}_{j}^{\prime} \leq \widetilde{a}_{j}$, then $E\left(\operatorname{ITrFLWA}\left(\widetilde{a}_{1}^{\prime}, \widetilde{a}_{2}^{\prime}, \ldots, \widetilde{a}_{n}^{\prime}\right)\right) \leq$ $\operatorname{E}\left(\operatorname{ITrFLWA}\left(\widetilde{a}_{1}, \widetilde{a}_{2}, \ldots, \widetilde{a}_{n}\right)\right)$,

$\operatorname{ITrFLWA}\left(\widetilde{a}_{1}^{\prime}, \widetilde{a}_{2}^{\prime}, \ldots, \widetilde{a}_{n}^{\prime}\right) \leq \operatorname{ITrFLWA}\left(\widetilde{a}_{1}, \widetilde{a}_{2}, \ldots, \widetilde{a}_{n}\right)$

Theorem 3.3. (Idempotency) Let $\widetilde{a}_{j}=\widetilde{a}$, for all $j=1,2, \ldots, n$, then

$$
\operatorname{ITrFLWA}\left(\widetilde{a}_{1}, \widetilde{a}_{2}, \ldots, \widetilde{a}_{n}\right)=\widetilde{a}
$$

Proof. Since $\widetilde{a}_{j}=\widetilde{a}$, thus

$$
\begin{aligned}
& \operatorname{ITrFLWA}\left(\widetilde{a}_{1}, \widetilde{a}_{2}, \ldots, \widetilde{a}_{n}\right) \\
& =\left\langle\left[ s_{\sum_{j=1}^{n} \omega_{j} \times \alpha\left(\widetilde{a}_{j}\right)}, s_{\sum_{j=1}^{n} \omega_{j} \times \beta\left(\widetilde{a}_{j}\right)},\right.\right. \\
& \left.s_{\sum_{j=1}^{n} \omega_{j} \times \theta\left(\widetilde{a}_{j}\right)}, s_{\sum_{j=1}^{n} \omega_{j} \times \tau\left(\widetilde{a}_{j}\right)}\right], \\
& \left.\left(1-\prod_{j=1}^{n}\left(1-u\left(\widetilde{a}_{j}\right)\right)\right)^{\omega_{j}}, \prod_{j=1}^{n}\left(v\left(\widetilde{a}_{j}\right)\right)^{\omega_{j}}\right\rangle \\
& =\left\langle\left[ s_{\sum_{j=1}^{n} \omega_{j} \times \alpha(\widetilde{a})}, s_{\sum_{j=1}^{n} \omega_{j} \times \beta(\widetilde{a})},\right.\right. \\
& s_{\sum_{j=1}^{n} \omega_{j} \times \theta(\widetilde{a})}, s_{\left.\sum_{j=1}^{n} \omega_{j} \times \tau(\widetilde{a})\right]}, \\
& \left.(1-(1-u(\widetilde{a})))^{\sum_{j=1}^{n} \omega_{j}},(v(\widetilde{a}))^{\sum_{j=1}^{n} \omega_{j}}\right\rangle \\
& =\left\langle\left[s_{\alpha(\widetilde{a})}, s_{\beta(\widetilde{a})}, s_{\theta(\widetilde{a})}, s_{\tau(\widetilde{a})}\right],(u(\widetilde{a}), v(\widetilde{a}))\right\rangle \\
& =\widetilde{a}
\end{aligned}
$$

Theorem 3.4. (Boundedness) Let $\widetilde{a}_{\text {min }}=\min _{1 \leq j \leq n}\left\{\widetilde{a}_{j}\right\}$ and $\widetilde{a}_{\max }=\max _{1 \leq j \leq n}\left\{\widetilde{a}_{j}\right\}$, then

$$
\widetilde{a}_{\min } \leq \operatorname{ITrFLWA}\left(\widetilde{a}_{1}, \widetilde{a}_{2}, \ldots, \widetilde{a}_{n}\right) \leq \widetilde{a}_{\max }
$$

Proof. Since $\widetilde{a}_{\min } \leq \widetilde{a} \leq \widetilde{a}_{\max }$, then

$$
\sum_{j=1}^{n} \omega_{j} \widetilde{a}_{m i n} \leq \sum_{j=1}^{n} \omega_{j} \widetilde{a}_{j} \leq \sum_{j=1}^{n} \omega_{j} \widetilde{a}_{\max }
$$

thus, $\widetilde{a}_{\min } \leq \operatorname{ITrF} \operatorname{LWA}\left(\widetilde{a}_{1}, \widetilde{a}_{2}, \ldots, \widetilde{a}_{n}\right) \leq \widetilde{a}_{\max }$

\section{Definition}

3.2.

Let $\widetilde{a}_{j}=\left\langle\left[s_{\alpha\left(\widetilde{a}_{j}\right)}, s_{\beta\left(\widetilde{a}_{j}\right)}, s_{\theta\left(\widetilde{a}_{j}\right)}, s_{\tau\left(\widetilde{a}_{j}\right)}\right],\left(u\left(\widetilde{a}_{j}\right), v\left(\widetilde{a}_{j}\right)\right)\right\rangle$ $(j=1,2, \ldots, n)$ be a collection of the ITrFLNs. The intuitionistic trapezoid fuzzy linguistic ordered weighted average (ITrFLOWA) operator can be defined as follows, and ITrFLOWA: $\Omega^{n} \rightarrow \Omega$ :

$$
\operatorname{ITrFLOWA}\left(\widetilde{a}_{1}, \widetilde{a}_{2}, \ldots, \widetilde{a}_{n}\right)=\sum_{j=1}^{n} w_{j} \widetilde{a}_{\sigma(j)}
$$

where $\Omega$ is the set of all intuitionistic trapezoid fuzzy linguistic numbers, and $w=\left(w_{1}, w_{2}, \ldots, w_{n}\right)^{T}$ is an associated weight vector with ITrFLOWA, such that $w_{j} \in[0,1] \quad(j=1,2, \ldots, n), \quad \sum_{j=1}^{n} w_{j}=1$. $(\sigma(1), \sigma(2), \ldots \sigma(n))$ is a permutation of $(1,2, \ldots, n)$ such that $\widetilde{a}_{\sigma(j-1)} \geq \widetilde{a}_{\sigma(j)}$ for all $j=2,3, \ldots, n . w_{j}$ is decided only by the $j$-th position in the aggregation process. Therefore, $w$ can also be called the position-weighted vector.

According to the method of determining position-weighted vector proposed in [24], $w$ can be calculated by the combination number. The calculation formula is as follows:

$$
w_{i+1}=\frac{c_{n-1}^{i}}{2^{n-1}},(i=0,1, \ldots, n-1)
$$

where the combination number $c_{n-1}^{i}$ can be denoted as $c_{n-1}^{i}=\frac{(n-1) !}{i !(n-i-1) !}$

Theorem 3.5. (Monotonicity) Let $\left(\widetilde{a}_{1}, \widetilde{a}_{2}, \ldots, \widetilde{a}_{n}\right)$ and $\left(\widetilde{a}_{1}^{\prime}, \widetilde{a}_{2}^{\prime}, \ldots, \widetilde{a}_{n}^{\prime}\right)$ be two collections of ITrFLNs. For all $j=1,2, \ldots, n$, if $\widetilde{a}_{j}^{\prime} \leq \widetilde{a}_{j}$, then

$$
\operatorname{ITrFLOWA}\left(\widetilde{a}_{1}, \widetilde{a}_{2}, \ldots, \widetilde{a}_{n}\right) \leq \operatorname{ITrFLOWA}\left(\widetilde{a}_{1}, \widetilde{a}_{2}, \ldots, \widetilde{a}_{n}\right)
$$

Theorem 3.6. (Idempotency) Let $\widetilde{a}_{j}=\widetilde{a}$, for all $j=1,2, \ldots, n$, then

$$
\operatorname{ITrFLOWA}\left(\widetilde{a}_{1}, \widetilde{a}_{2}, \ldots, \widetilde{a}_{n}\right)=\sum_{j=1}^{n} w_{j} \widetilde{a}_{j}=\widetilde{a}
$$

Theorem 3.7. (Boundedness) Let $\widetilde{a}_{\text {min }}=\min _{1 \leq j \leq n}\left\{\widetilde{a}_{j}\right\}$ and $\widetilde{a}_{\max }=\max _{1 \leq j \leq n}\left\{\widetilde{a}_{j}\right\}$, then

$$
\widetilde{a}_{\text {min }} \leq \operatorname{ITrFLOWA}\left(\widetilde{a}_{1}, \widetilde{a}_{2}, \ldots, \widetilde{a}_{n}\right) \leq \widetilde{a}_{\max }
$$

Theorems 3.5-3.7 can be easily proven similar to Theorems 3.2-3.4, so the proofs are omitted. 
Theorem 3.8. (Commutativity) If $\left(\widetilde{a}_{1}^{\prime}, \widetilde{a}_{2}^{\prime}, \ldots, \widetilde{a}_{n}^{\prime}\right)$ is any permutation of $\left(\widetilde{a}_{1}, \widetilde{a}_{2}, \ldots, \widetilde{a}_{n}\right)$, then

$$
\operatorname{ITrFLOWA}\left(\widetilde{a}_{1}, \widetilde{a}_{2}, \ldots, \widetilde{a}_{n}\right)=\operatorname{ITrFLOWA}\left(\widetilde{a}_{1}, \widetilde{a}_{2}, \ldots, \widetilde{a}_{n}\right)
$$

Proof. Since $\left(\widetilde{a}_{1}^{\prime}, \widetilde{a}_{2}^{\prime}, \ldots, \widetilde{a}_{n}\right)$ is any permutation of $\left(\widetilde{a}_{1}, \widetilde{a}_{2}, \ldots, \widetilde{a}_{n}\right)$, then

$$
\begin{aligned}
\sum_{j=1}^{n} w_{j} \times \alpha\left(\widetilde{a}_{\sigma(j)}\right) & =\sum_{j=1}^{n} w_{j} \times \alpha\left(\widetilde{a}_{\sigma(j)}\right) \\
\sum_{j=1}^{n} w_{j} \times \beta\left(\widetilde{a}_{\sigma(j)}\right) & =\sum_{j=1}^{n} w_{j} \times \beta\left(\widetilde{a}_{\sigma(j)}\right) \\
\sum_{j=1}^{n} w_{j} \times \theta\left(\widetilde{a}_{\sigma(j)}\right) & =\sum_{j=1}^{n} w_{j} \times \theta\left(\widetilde{a}_{\sigma(j)}\right) \\
\sum_{j=1}^{n} w_{j} \times \tau\left(\widetilde{a}_{\sigma(j)}\right) & =\sum_{j=1}^{n} w_{j} \times \tau\left(\widetilde{a}_{\sigma(j)}\right) \\
1-\prod_{j=1}^{n}\left(1-u\left(\widetilde{a}_{\sigma(j)}\right)\right)^{w_{j}} & =1-\prod_{j=1}^{n}\left(1-u\left(\widetilde{a}_{\sigma(j)}\right)\right)^{w_{j}} \\
\prod_{j=1}^{n}\left(v\left(\widetilde{a}_{\sigma(j)}\right)\right)^{w_{j}} & =\prod_{j=1}^{n}\left(v\left(\widetilde{a}_{\sigma(j)}\right)\right)^{w_{j}}
\end{aligned}
$$

thus,

$\operatorname{ITrFLOWA}\left(\widetilde{a}_{1}, \widetilde{a}_{2}, \ldots, \widetilde{a}_{n}\right)=\operatorname{ITrFLOWA}\left(\widetilde{a}_{1}^{\prime}, \widetilde{a}_{2}^{\prime}, \ldots, \widetilde{a}_{n}^{\prime}\right)$.

Definition

3.3. $\widetilde{a}_{j}=\left\langle\left[s_{\alpha\left(\widetilde{a}_{j}\right)}, s_{\beta\left(\widetilde{a}_{j}\right)}, s_{\theta\left(\widetilde{a}_{j}\right)}, s_{\tau\left(\widetilde{a}_{j}\right)}\right],\left(u\left(\widetilde{a}_{j}\right), v\left(\widetilde{a}_{j}\right)\right)\right\rangle$ $(j=1,2, \ldots, n)$ be a collection of the ITrFLNs. The intuitionistic trapezoid fuzzy linguistic hybrid weighted average (ITrFLHWA) operator can be defined as follows, and ITrFLHWA: $\Omega^{n} \rightarrow \Omega$ :

$$
\operatorname{ITrFLHWA}\left(\widetilde{a}_{1}, \widetilde{a}_{2}, \ldots, \widetilde{a}_{n}\right)=\sum_{j=1}^{n} w_{j} \widetilde{b}_{\sigma(j)}
$$

where $\Omega$ is the set of all intuitionistic trapezoid fuzzy linguistic numbers, $w=\left(w_{1}, w_{2}, \ldots, w_{n}\right)^{T}$ is an associated weight vector with ITrFLHWA, such that $w_{j} \in[0,1]$ and $\sum_{j=1}^{n} w_{j}=1 . \quad \tilde{b}_{j}=n \omega_{j} \tilde{a}_{j} \quad j=1,2, \ldots, n$, $\left(\widetilde{b}_{\sigma(1)}, \widetilde{b}_{\sigma(2)}, \ldots, \widetilde{b}_{\sigma(n)}\right)$ is a permutation of $\left(\widetilde{b}_{1}, \widetilde{b}_{2}, \ldots, \widetilde{b}_{n}\right)$, such that $\widetilde{b}_{\sigma(j-1)} \geq \widetilde{b}_{\sigma(j)}$ for $j=2,3, \ldots, n$. $\omega=\left(\omega_{1}, \omega_{2}, \ldots, \omega_{n}\right)^{T}$ is the weight vector of $\tilde{a}_{j}$ $(j=1,2, \ldots, n), \omega_{j} \in[0,1], \sum_{j=1}^{n} \omega_{j}=1$ and $n$ is the balancing coefficient.

According to the operations of ITrFLNs, Eq. (21) can be transformed into the following form.

$$
\begin{aligned}
& \operatorname{ITrFLHWA}\left(\widetilde{a}_{1}, \widetilde{a}_{2}, \ldots, \widetilde{a}_{n}\right) \\
= & \left\langle\left[ s_{\sum_{j=1}^{n} w_{j} \times \alpha\left(\widetilde{b}_{\sigma(j)}\right)}, s_{\sum_{j=1}^{n} w_{j} \times \beta\left(\widetilde{b}_{\sigma(j)}\right)},\right.\right. \\
& \left.s_{\sum_{j=1}^{n} w_{j} \times \theta\left(\widetilde{b}_{\sigma(j)}\right)}, s_{\sum_{j=1}^{n} w_{j} \times \tau\left(\widetilde{b}_{\sigma(j)}\right)}\right], \\
& \left.\left(1-\prod_{j=1}^{n}\left(1-u\left(\widetilde{b}_{\sigma(j)}\right)\right)\right)^{w_{j}}, \prod_{j=1}^{n}\left(v\left(\widetilde{b}_{\sigma(j)}\right)\right)^{w_{j}}\right\rangle
\end{aligned}
$$

Two special cases of the ITrFLHWA operator are as follows:
1.When $w=\left(\frac{1}{n}, \frac{1}{n}, \ldots, \frac{1}{n}\right)^{T}$, the ITrFLHWA operator reduces to the ITrFLWA operator in Eq. (10).

2. When $\omega=\left(\frac{1}{n}, \frac{1}{n}, \ldots, \frac{1}{n}\right)^{T}$, the ITrFLHWA operator reduces to the ITrFLOWA operator in Eq. (15).

\section{Two approaches for multi-attribute group decision making based on intuitionistic trapezoid fuzzy linguistic aggregation operators}

For the multi-attribute group decision making problems in this paper, weights of both the attribute and the decision makers take the form of real numbers, and the attribute preference values take the form of intuitionistic trapezoid fuzzy linguistic variables. Therefore, we shall develop approaches to group decision making based on intuitionistic trapezoid fuzzy linguistic preference information.

Let $A=\left\{A_{1}, A_{2}, \ldots, A_{m}\right\}$ be a discrete set of alternatives, $C=\left\{C_{1}, C_{2}, \ldots, C_{n}\right\}$ be a set of attributes, and $\omega=\left(\omega_{1}, \omega_{2}, \ldots, \omega_{n}\right)^{T}$ be the weight vector of the attributes, such that $\omega_{j} \in[0,1](j=1,2, \ldots, n)$, and $\sum_{j=1}^{n} \omega_{j}=1 . D M=\left\{D M_{1}, D M_{2}, \ldots, D M_{k}\right\}$ is the set of decision makers. $R_{f}=\left[\widetilde{a}_{i j}^{f}\right]_{m \times n}(f=1,2, \ldots, k)$ is the decision matrix given by the decision maker $D M_{f}$, where $\tilde{a}_{i j}^{f}=\left\langle\left[s_{\alpha\left(\widetilde{a}_{i j}^{f}\right)}, s_{\beta\left(\widetilde{a}_{i j}^{f}\right)}, s_{\theta\left(\widetilde{a}_{i j}^{f}\right)}, s_{\tau\left(\widetilde{a}_{i j}^{f}\right)}\right],\left(u\left(\widetilde{a}_{i j}^{f}\right), v\left(\widetilde{a}_{i j}^{f}\right)\right)\right\rangle$ denotes the evaluation on alternative $A_{i}$ with respect to attribute $C_{j}$ given by the decision maker $D M_{f}$, and it takes the form of intuitionistic trapezoid fuzzy linguistic number.

\section{Approach 1}

If the weight vector of the decision makers is known and defined as $w=\left(w_{1}, w_{2}, \ldots, w_{k}\right)^{T}$, we select the best alternative by the following steps.

Step 1: For each decision maker $D M_{f}(f=1,2, \ldots, k)$, utilize the weight vector of the attributes $\omega=\left(\omega_{1}, \omega_{2}, \ldots, \omega_{n}\right)^{T}$ and ITrFLWA operator in Eq. (10) to calculate the comprehensive attribute preference value $\widetilde{a}_{i}^{f}$ of each alternative determined by the $f$-th decision maker, respectively.

$\widetilde{a}_{i}^{f}=\operatorname{ITrFLWA}\left(\widetilde{a}_{i 1}^{f}, \widetilde{a}_{i 2}^{f}, \ldots, \widetilde{a}_{i n}^{f}\right)=\sum_{j=1}^{n} w_{j} \widetilde{a}_{i j}^{f},(i=1,2, \ldots, m)$

Step 2: For each alternative, calculate the collective overall preference values $Z_{i}$ of each alternative determined by all decision makers by Eq. (24).

$$
Z_{i}=\sum_{f=1}^{k} w_{f} \widetilde{a}_{i}^{f},(i=1,2, \ldots, m)
$$

Step 3: Utilize the expected function in Eq. (7) to calculate the expected values $E\left(Z_{i}\right)(i=1,2, \ldots, m)$ of the 
collective overall preference values $Z_{i}$. If there is no difference between two expected values $E\left(Z_{i}\right)$ and $E\left(Z_{j}\right)$, then we need to calculate the accuracy values $H\left(Z_{i}\right)$ and $H\left(Z_{i}\right)$ of value $Z_{i}$ and $Z_{j}$ by Eq. (8).

Step 4: Rank these alternatives and select the best one by the values of $E\left(Z_{i}\right)$ and $H\left(Z_{i}\right)$.

\section{Approach 2}

If the weight vector of the decision makers is unknown, we select the best alternative by the following steps.

Step 1: See Step 1 in Approach 1.

Step 2: Calculate the weight vector $w=\left(w_{1}, w_{2}, \ldots, w_{n}\right)^{T}$ of the decision maker $D M_{f}(f=1,2, \ldots, k)$ by Eq. (16) at first. Then utilize ITrFLOWA operator to calculate $Z_{i}$ by Eq. (25)

$$
Z_{i}=\sum_{f=1}^{k} w_{f} \widetilde{a}_{i}^{\sigma(f)},(i=1,2, \ldots, m)
$$

where $\left(\widetilde{a}_{i}^{\sigma(1)}, \widetilde{a}_{i}^{\sigma(2)}, \ldots, \widetilde{a}_{i}^{\sigma(k)}\right)$ is a permutation of $\left(\widetilde{a}_{i}^{1}, \widetilde{a}_{i}^{2}, \ldots, \widetilde{a}_{i}^{k}\right)$, such that $\widetilde{a}_{i}^{\sigma(f-1)} \geq \widetilde{a}_{i}^{\sigma(f)}(f=2,3, \ldots, k)$.

Step 3: See Step 3 in Approach 1.

Step 4: See Step 4 in Approach 1.

\section{An illustrative example}

A serious public health event happens in one city of China recently, emergency management center of the government wants to select a most appropriate emergency logistics supplier in order to implement rescue activities immediately, reducing the economic losses and rescue cost maximally. There are five suppliers in the city: $\left\{A_{1}, A_{2}, A_{3}, A_{4}, A_{5}\right\}$. The emergency management center must make a decision according to the following four attributes with the attribute weight vector $\omega=(0.3,0.4,0.2,0.1)^{T}$ : (1) $C_{1}$ is the emergency resource supply capacity; (2) $C_{2}$ is the transportation speed; (3) $C_{3}$ is the transportation distance; (4) $C_{4}$ is the transportation risk.

Four experts $\left(D M_{1}, D M_{2}, D M_{3}, D M_{4}\right)$ are invited to evaluate the suppliers with respect to each attribute by using the predefined linguistic term set $S=\left\{s_{0}\right.$ (extremely low); $s_{1}$ (very low); $s_{2}$ (low); $s_{3}$ (medium); $s_{4}$ (high); $s_{5}$ (very high); $s_{6}$ (extremely high) $\}$, and the weight vector of the decision makers is $w=(0.25,0.20,0.30,0.25)^{T}$. The intuitionistic trapezoid fuzzy linguistic decision matrices $R_{f}=\left[\widetilde{a}_{i j}^{f}\right]_{5 \times 4}$ $(f=1,2,3,4)$ are constructed as shown in [Tables 1-4].

Step 1: Utilize the weight vector of the attributes $\omega=(0.3,0.4,0.2,0.1)^{T}$ to calculate the comprehensive attribute preference values $\widetilde{a}_{i}^{f}$ $(i=1,2,3,4,5 ; f=1,2,3,4)$ by Eq. (23), which are shown in the following, respectively.

$$
\begin{aligned}
& \tilde{a}_{1}^{1}=\left\langle\left[s_{1.8}, s_{3.4}, s_{4.5}, s_{5.5}\right],(0.6378,0.3051)\right\rangle ; \\
& \tilde{a}_{2}^{1}=\left\langle\left[s_{1.3}, s_{2.3}, s_{4.4}, s_{5.4}\right],(0.6536,0.2408)\right\rangle ; \\
& \tilde{a}_{3}^{1}=\left\langle\left[s_{0.8}, s_{2.2}, s_{3.9}, s_{5.1}\right],(0.6607,0.2048)\right\rangle ; \\
& \tilde{a}_{4}^{1}=\left\langle\left[s_{1.4}, s_{2.8}, s_{3.8}, s_{5.1}\right],(0.6102,0.1762)\right\rangle ; \\
& \tilde{a}_{5}^{1}=\left\langle\left[s_{1.5}, s_{2.5}, s_{4.5}, s_{5.5}\right],(0.6378,0.2521)\right\rangle ; \\
& \tilde{a}_{1}^{2}=\left\langle\left[s_{1.1}, s_{2.1}, s_{4.4}, s_{5.4}\right],(0.6005,0.3170)\right\rangle ; \\
& \tilde{a}_{2}^{2}=\left\langle\left[s_{2.1}, s_{3.2}, s_{4.5}, s_{5.7}\right],(0.5837,0.2844)\right\rangle ; \\
& \widetilde{a}_{3}^{2}=\left\langle\left[s_{0.7}, s_{1.7}, s_{4.6}, s_{5.6}\right],(0.6536,0.2169)\right\rangle ; \\
& \tilde{a}_{4}^{2}=\left\langle\left[s_{1.5}, s_{3.3}, s_{4.6}, s_{5.6}\right],(0.6969,0.2564)\right\rangle ; \\
& \tilde{a}_{5}^{2}=\left\langle\left[s_{1.8}, s_{3.1}, s_{4.3}, s_{5.3}\right],(0.6536,0.2479)\right\rangle ; \\
& \tilde{a}_{1}^{3}=\left\langle\left[s_{1.4}, s_{2.4}, s_{4.4}, s_{5.7}\right],(0.6536,0.1231)\right\rangle ; \\
& \tilde{a}_{2}^{3}=\left\langle\left[s_{1.5}, s_{3.1}, s_{4.7}, s_{5.9}\right],(0.6751,0.2024)\right\rangle ; \\
& \tilde{a}_{3}^{3}=\left\langle\left[s_{1.6}, s_{2.7}, s_{4.1}, s_{5.5}\right],(0.6536,0.2048)\right\rangle ; \\
& \tilde{a}_{4}^{3}=\left\langle\left[s_{1.7}, s_{2.7}, s_{4.1}, s_{5.1}\right],(0.5557,0.2885)\right\rangle ; \\
& \tilde{a}_{5}^{3}=\left\langle\left[s_{1.7}, s_{2.9}, s_{4.0}, s_{5.4}\right],(0.6534,0.2334)\right\rangle ; \\
& \tilde{a}_{1}^{4}=\left\langle\left[s_{1.5}, s_{2.5}, s_{4.5}, s_{5.5}\right],(0.6435,0.2352)\right\rangle ; \\
& \tilde{a}_{2}^{4}=\left\langle\left[s_{1.6}, s_{2.8}, s_{4.2}, s_{5.5}\right],(0.6634,0.1712)\right\rangle ; \\
& \tilde{a}_{3}^{4}=\left\langle\left[s_{1.2}, s_{1.8}, s_{4.5}, s_{5.5}\right],(0.6098,0.2595)\right\rangle ; \\
& \tilde{a}_{4}^{4}=\left\langle\left[s_{1.8}, s_{2.8}, s_{4.4}, s_{5.5}\right],(0.6212,0.3092)\right\rangle ; \\
& \widetilde{a}_{5}^{4}=\left\langle\left[s_{1.4}, s_{2.7}, s_{3.0}, s_{5.3}\right],(0.6534,0.2319)\right\rangle
\end{aligned}
$$

Step 2: Calculate the collective overall preference value $Z_{i}$ of each alternative by Eq. (24). The collective overall preference values $Z_{i}$ are shown in the following.

$$
\begin{aligned}
& Z_{1}=\left\langle\left[s_{1.465}, s_{2.615}, s_{4.450}, s_{5.540}\right],(0.6370,0.2194)\right\rangle ; \\
& Z_{2}=\left\langle\left[s_{1.595}, s_{2.845}, s_{4.460}, s_{5.635}\right],(0.6500,0.2170)\right\rangle ; \\
& Z_{3}=\left\langle\left[s_{1.120}, s_{2.400}, s_{4.250}, s_{5.420}\right],(0.6449,0.2198)\right\rangle ; \\
& Z_{4}=\left\langle\left[s_{1.610}, s_{2.870}, s_{4.200}, s_{5.300}\right],(0.6172,0.2535)\right\rangle ; \\
& Z_{5}=\left\langle\left[s_{1.595}, s_{2.790}, s_{4.160}, s_{5.380}\right],(0.6496,0.2404)\right\rangle .
\end{aligned}
$$

Step 3: Utilize the expected function in Eq. (7) to calculate the expected values $E\left(Z_{i}\right)(i=1,2,3,4,5)$ of collective overall preference values $Z_{i}$

$$
\begin{aligned}
& E\left(Z_{1}\right)=s_{2.4931} ; E\left(Z_{2}\right)=s_{2.6036} ; E\left(Z_{3}\right)=s_{2.3498} ; \\
& E\left(Z_{4}\right)=s_{2.3832} ; E\left(Z_{5}\right)=s_{2.4529} .
\end{aligned}
$$

Step 4: Rank these alternatives and select the best one by the value of $E\left(Z_{i}\right)$.

$A_{2} \succ A_{1} \succ A_{5} \succ A_{4} \succ A_{3}$

Therefore, the best alternative is $A_{2}$.

If the weight vector of the decision makers is unknown, we select the best alternative by the following steps.

Step 1: See Step 1 in above example.

Step 2: If the weight vector of the decision makers is unknown, it is necessary to calculate the weight vector 
Table 1: Intuitionistic trapezoid fuzzy linguistic decision matrix $R_{1}$ given by $D M_{1}$

\begin{tabular}{ccccc}
\hline Alternatives & $C_{1}$ & $C_{2}$ & $C_{3}$ & $C_{4}$ \\
\hline$A_{1}$ & $\left\langle\left[s_{2}, s_{3}, s_{4}, s_{5}\right],(0.6,0.3)\right\rangle$ & $\left\langle\left[s_{2}, s_{4}, s_{5}, s_{6}\right],(0.7,0.3)\right\rangle$ & $\left\langle\left[s_{1}, s_{3}, s_{4}, s_{5}\right],(0.5,0.4)\right\rangle$ & $\left\langle\left[s_{2}, s_{3}, s_{5}, s_{6}\right],(0.7,0.2)\right\rangle$ \\
$A_{2}$ & $\left\langle\left[s_{1}, s_{2}, s_{4}, s_{5}\right],(0.7,0.3)\right\rangle$ & $\left\langle\left[s_{2}, s_{3}, s_{5}, s_{6}\right],(0.6,0.3)\right\rangle$ & $\left\langle\left[s_{0}, s_{1}, s_{4}, s_{5}\right],(0.7,0.1)\right\rangle$ & $\left\langle\left[s_{2}, s_{3}, s_{4}, s_{5}\right],(0.6,0.3)\right\rangle$ \\
$A_{3}$ & $\left\langle\left[s_{0}, s_{2}, s_{4}, s_{5}\right],(0.7,0.2)\right\rangle$ & $\left\langle\left[s_{1}, s_{2}, s_{4}, s_{5}\right],(0.5,0.3)\right\rangle$ & $\left\langle\left[s_{1}, s_{2}, s_{3}, s_{5}\right],(0.8,0.1)\right\rangle$ & $\left\langle\left[s_{2}, s_{4}, s_{5}, s_{6}\right],(0.7,0.2)\right\rangle$ \\
$A_{4}$ & $\left\langle\left[s_{1}, s_{2}, s_{3}, s_{5}\right],(0.5,0.2)\right\rangle$ & $\left\langle\left[s_{1}, s_{3}, s_{4}, s_{5}\right],(0.7,0.1)\right\rangle$ & $\left\langle\left[s_{2}, s_{3}, s_{4}, s_{5}\right],(0.6,0.3)\right\rangle$ & $\left\langle\left[s_{3}, s_{4}, s_{5}, s_{6}\right],(0.5,0.4)\right\rangle$ \\
$A_{5}$ & $\left\langle\left[s_{2}, s_{3}, s_{5}, s_{6}\right],(0.6,0.2)\right\rangle$ & $\left\langle\left[s_{2}, s_{3}, s_{4}, s_{5}\right],(0.7,0.3)\right\rangle$ & $\left\langle\left[s_{0}, s_{1}, s_{5}, s_{6}\right],(0.5,0.4)\right\rangle$ & $\left\langle\left[s_{1}, s_{2}, s_{4}, s_{5}\right],(0.7,0.1)\right\rangle$ \\
\hline
\end{tabular}

Table 2: Intuitionistic trapezoid fuzzy linguistic decision matrix $R_{2}$ given by $D M_{2}$

\begin{tabular}{ccccc}
\hline Alternatives & $C_{1}$ & $C_{2}$ & $C_{3}$ & $C_{4}$ \\
\hline$A_{1}$ & $\left\langle\left[s_{0}, s_{1}, s_{5}, s_{6}\right],(0.7,0.2)\right\rangle$ & $\left\langle\left[s_{1}, s_{2}, s_{4}, s_{5}\right],(0.6,0.4)\right\rangle$ & $\left\langle\left[s_{2}, s_{3}, s_{4}, s_{5}\right],(0.5,0.5)\right\rangle$ & $\left\langle\left[s_{3}, s_{4}, s_{5}, s_{6}\right],(0.4,0.2)\right\rangle$ \\
$A_{2}$ & $\left\langle\left[s_{2}, s_{3}, s_{5}, s_{6}\right],(0.5,0.3)\right\rangle$ & $\left\langle\left[s_{3}, s_{4}, s_{5}, s_{6}\right],(0.5,0.4)\right\rangle$ & $\left\langle\left[s_{1}, s_{2}, s_{3}, s_{5}\right],(0.8,0.1)\right\rangle$ & $\left\langle\left[s_{1}, s_{3}, s_{4}, s_{5}\right],(0.5,0.5)\right\rangle$ \\
$A_{3}$ & $\left\langle\left[s_{0}, s_{1}, s_{4}, s_{5}\right],(0.6,0.2)\right\rangle$ & $\left\langle\left[s_{0}, s_{1}, s_{5}, s_{6}\right],(0.7,0.2)\right\rangle$ & $\left\langle\left[s_{3}, s_{4}, s_{5}, s_{6}\right],(0.6,0.3)\right\rangle$ & $\left\langle\left[s_{1}, s_{2}, s_{4}, s_{5}\right],(0.7,0.2)\right\rangle$ \\
$A_{4}$ & $\left\langle\left[s_{1}, s_{3}, s_{4}, s_{5}\right],(0.6,0.4)\right\rangle$ & $\left\langle\left[s_{2}, s_{4}, s_{5}, s_{6}\right],(0.8,0.2)\right\rangle$ & $\left\langle\left[s_{2}, s_{3}, s_{5}, s_{6}\right],(0.6,0.2)\right\rangle$ & $\left\langle\left[s_{0}, s_{2}, s_{4}, s_{5}\right],(0.6,0.3)\right\rangle$ \\
$A_{5}$ & $\left\langle\left[s_{2}, s_{4}, s_{5}, s_{6}\right],(0.7,0.3)\right\rangle$ & $\left\langle\left[s_{2}, s_{3}, s_{4}, s_{5}\right],(0.6,0.3)\right\rangle$ & $\left\langle\left[s_{1}, s_{2}, s_{4}, s_{5}\right],(0.7,0.1)\right\rangle$ & $\left\langle\left[s_{2}, s_{3}, s_{4}, s_{5}\right],(0.6,0.4)\right\rangle$ \\
\hline
\end{tabular}

Table 3: Intuitionistic trapezoid fuzzy linguistic decision matrix $R_{3}$ given by $D M_{3}$

\begin{tabular}{ccccc}
\hline Alternatives & \multicolumn{1}{c}{$C_{1}$} & $C_{2}$ & $C_{3}$ & $C_{4}$ \\
\hline$A_{1}$ & $\left\langle\left[s_{0}, s_{1}, s_{4}, s_{6}\right],(0.7,0.1)\right\rangle$ & $\left\langle\left[s_{3}, s_{4}, s_{5}, s_{6}\right],(0.6,0.1)\right\rangle$ & $\left\langle\left[s_{1}, s_{2}, s_{4}, s_{5}\right],(0.7,0.2)\right\rangle$ & $\left\langle\left[s_{0}, s_{1}, s_{4}, s_{5}\right],(0.6,0.2)\right\rangle$ \\
$A_{2}$ & $\left\langle\left[s_{2}, s_{4}, s_{5}, s_{6}\right],(0.8,0.2)\right\rangle$ & $\left\langle\left[s_{2}, s_{3}, s_{5}, s_{6}\right],(0.6,0.2)\right\rangle$ & $\left\langle\left[s_{0}, s_{2}, s_{4}, s_{6}\right],(0.6,0.3)\right\rangle$ & $\left\langle\left[s_{1}, s_{3}, s_{4}, s_{5}\right],(0.6,0.1)\right\rangle$ \\
$A_{3}$ & $\left\langle\left[s_{2}, s_{3}, s_{4}, s_{5}\right],(0.6,0.3)\right\rangle$ & $\left\langle\left[s_{1}, s_{2}, s_{4}, s_{6}\right],(0.7,0.1)\right\rangle$ & $\left\langle\left[s_{2}, s_{3}, s_{4}, s_{5}\right],(0.6,0.4)\right\rangle$ & $\left\langle\left[s_{2}, s_{4}, s_{5}, s_{6}\right],(0.7,0.3)\right\rangle$ \\
$A_{4}$ & $\left\langle\left[s_{1}, s_{2}, s_{4}, s_{5}\right],(0.6,0.2)\right\rangle$ & $\left\langle\left[s_{2}, s_{3}, s_{4}, s_{5}\right],(0.5,0.5)\right\rangle$ & $\left\langle\left[s_{3}, s_{4}, s_{5}, s_{6}\right],(0.5,0.2)\right\rangle$ & $\left\langle\left[s_{0}, s_{1}, s_{3}, s_{4}\right],(0.7,0.2)\right\rangle$ \\
$A_{5}$ & $\left\langle\left[s_{3}, s_{4}, s_{5}, s_{6}\right],(0.5,0.4)\right\rangle$ & $\left\langle\left[s_{1}, s_{2}, s_{3}, s_{5}\right],(0.8,0.1)\right\rangle$ & $\left\langle\left[s_{1}, s_{3}, s_{4}, s_{5}\right],(0.5,0.5)\right\rangle$ & $\left\langle\left[s_{2}, s_{3}, s_{5}, s_{6}\right],(0.5,0.3)\right\rangle$ \\
\hline
\end{tabular}

Table 4: Intuitionistic trapezoid fuzzy linguistic decision matrix $R_{4}$ given by $D M_{4}$

\begin{tabular}{|c|c|c|c|c|}
\hline Alternatives & $C_{1}$ & $C_{2}$ & $C_{3}$ & $C_{4}$ \\
\hline$A_{1}$ & $\left\langle\left[s_{1}, s_{2}, s_{4}, s_{5}\right],(0.7,0.2)\right\rangle$ & $\left\langle\left[s_{3}, s_{4}, s_{5}, s_{6}\right],(0.6,0.3)\right\rangle$ & $\left\langle\left[s_{0}, s_{1}, s_{4}, s_{5}\right],(0.6,0.2)\right\rangle$ & $\left\langle\left[s_{0}, s_{1}, s_{5}, s_{6}\right],(0.7,0.2)\right\rangle$ \\
\hline$A_{2}$ & $\left\langle\left[s_{2}, s_{3}, s_{4}, s_{6}\right],(0.6,0.2)\right\rangle$ & $\left\langle\left[s_{1}, s_{2}, s_{4}, s_{5}\right],(0.7,0.1)\right\rangle$ & $\left\langle\left[s_{2}, s_{4}, s_{5}, s_{6}\right],(0.7,0.3)\right\rangle$ & $\left\langle\left[s_{2}, s_{3}, s_{4}, s_{5}\right],(0.6,0.3)\right\rangle$ \\
\hline$A_{3}$ & $\left\langle\left[s_{0}, s_{2}, s_{4}, s_{5}\right],(0.6,0.3)\right\rangle$ & $\left\langle\left[s_{2}, s_{3}, s_{5}, s_{6}\right],(0.6,0.2)\right\rangle$ & $\left\langle\left[s_{1}, s_{3}, s_{4}, s_{5}\right],(0.5,0.4)\right\rangle$ & $\left\langle\left[s_{2}, s_{4}, s_{5}, s_{6}\right],(0.8,0.2)\right\rangle$ \\
\hline$A_{4}$ & $\left\langle\left[s_{3}, s_{4}, s_{5}, s_{6}\right],(0.7,0.2)\right\rangle$ & $\left\langle\left[s_{2}, s_{3}, s_{4}, s_{5}\right],(0.5,0.5)\right\rangle$ & $\left\langle\left[s_{0}, s_{1}, s_{5}, s_{6}\right],(0.7,0.2)\right\rangle$ & $\left\langle\left[s_{1}, s_{2}, s_{3}, s_{5}\right],(0.6,0.4)\right\rangle$ \\
\hline$A_{5}$ & $\left\langle\left[s_{1}, s_{3}, s_{4}, s_{5}\right],(0.5,0.5)\right\rangle$ & $\left\langle\left[s_{1}, s_{2}, s_{3}, s_{5}\right],(0.8,0.1)\right\rangle$ & $\left\langle\left[s_{2}, s_{3}, s_{5}, s_{6}\right],(0.5,0.3)\right\rangle$ & $\left\langle\left[s_{3}, s_{4}, s_{5}, s_{6}\right],(0.5,0.4)\right\rangle$ \\
\hline
\end{tabular}

$w=\left(w_{1}, w_{2}, w_{3}, w_{4}\right)^{T}$ of decision makers by Eq. (16) at first.

$w_{1}=\frac{c_{3}^{0}}{2^{3}}=0.125, w_{1}=\frac{c_{3}^{1}}{2^{3}}=0.375$

$w_{1}=\frac{c_{3}^{2}}{2^{3}}=0.375, w_{1}=\frac{c_{3}^{3}}{2^{3}}=0.125$.

Then calculate the collective overall preference value $Z_{1}$ by Eq. (25), and the results are shown in the following.

$Z_{1}=\left\langle\left[s_{1.5500}, s_{2.7750}, s_{4.4750}, s_{5.5125}\right],(0.6375,0.2483)\right\rangle ;$

$Z_{2}=\left\langle\left[s_{1.7375}, s_{2.9250}, s_{4.4000}, s_{5.6125}\right],(0.6358,0.2207)\right\rangle ;$

$Z_{3}=\left\langle\left[s_{1.0125}, s_{2.3000}, s_{4.4125}, s_{5.4875}\right],(0.6387,0.2287)\right\rangle ;$

$Z_{4}=\left\langle\left[s_{1.6000}, s_{2.8500}, s_{4.1625}, s_{5.3125}\right],(0.6201,0.2425)\right\rangle ;$

$Z_{5}=\left\langle\left[s_{1.6000}, s_{2.7500}, s_{4.2125}, s_{5.4125}\right],(0.6477,0.2419)\right\rangle$.

Step 3: Calculate the expected values $E\left(Z_{i}\right)$ $i=(1,2,3,4,5)$ of collective overall preference values $Z_{i}$ by Eq. (7).

$E\left(Z_{1}\right)=s_{2.4855} ; E\left(Z_{2}\right)=s_{2.5959} ; E\left(Z_{3}\right)=s_{2.3288} ;$

$E\left(Z_{4}\right)=s_{2.3979} ; E\left(Z_{5}\right)=s_{2.4557}$.
Step 4: Rank these alternatives and select the best one by the value of $E\left(Z_{i}\right)$.

$A_{2} \succ A_{1} \succ A_{5} \succ A_{4} \succ A_{3}$

Therefore, the best alternative is $A_{2}$ as well.

\section{Conclusions}

In this paper, we study the multi-attribute group decision making (MAGDM) problems in which the attribute values provided by the decision makers take the form of intuitionistic trapezoid fuzzy linguistic information. We have developed some new intuitionistic trapezoid fuzzy linguistic aggregation operators, such as intuitionistic trapezoid fuzzy linguistic weighted average (ITrFLWA) operator, intuitionistic trapezoid fuzzy linguistic ordered weighted average (ITrFLOWA) operator and intuitionistic trapezoid fuzzy linguistic hybrid weighted average (ITrFLHWA) operator firstly. Then we have studied some desired properties of the developed operators, such as monotonicity, commutativity, idempotency and 
boundedness. Moreover, we have developed two approaches to deal with multi-attribute group decision making problems under intuitionistic trapezoid fuzzy linguistic information. If the weight vector of the decision makers is known, we develop an approach based on the ITrFLWA operator to aggregate all attribute preference values into comprehensive attribute preference values, and then derive the collective overall evaluation values of each alternative. On the other hand, if the weight vector of the decision makers is unknown, we develop another approach based on the ITrFLOWA to aggregate all the comprehensive attribute preference values into collective overall preference values and derive the overall evaluation values of each alternative. Finally, an illustrative example has been given to show the developed method. Apparently, our approaches are straightforward and have less loss of information both theoretically and practically, and can be applied to handle MAGDM problems in a flexible and objective manner under intuitionistic trapezoid fuzzy linguistic environment. It is worth noting that in addition to emergency logistics supplier selection problem, the proposed methodes are equally applicable to other management decision problems. In the future, we shall continue working in the extension and application of the developed operators.

\section{Acknowledgement}

This research is supported by Program for New Century Excellent Talents in University (NCET-13-0037), Natural Science Foundation of China (No. 70972007, 71271049) and Beijing Municipal Natural Science Foundation (No. 9102015).

\section{References}

[1] L. A. Zadeh, Fuzzy Sets, Information and Control, 8, 338-353 (1965).

[2] L. A. Zadeh, The concept of a linguistic variable and its application to approximate reasoning, Information sciences, 8, 199-249 (1975).

[3] F. Herrera, E. Herrera-Viedma and J. L. Verdegay, A model of consensus in group decision making under linguistic assessments, Fuzzy Sets and Systems, 78, 73-87 (1996).

[4] F. Herrera and L. Martłnez, A 2-Tuple Fuzzy Linguistic Representation Model for Computing with Words, IEEE Transactions on Fuzzy Systems, 8, 746752 (2000).

[5] J. Lin, J. B. Lan and Y. H. Lin, Multi-attribute group decision-making method based on the aggregation operators of interval 2-tuple linguistic information, Journal of Jilin Normal University, 1, 5-9 (2009).

[6] Z. S. Xu, Uncertain linguistic aggregation operators based approach to multiple attribute group decision making under uncertain linguistic environment, Information Sciences, 168, 171-184 (2004).

[7] H. M. Zhang, The multiattribute group decision making method based on aggregation operators with interval-valued 2-tuple linguistic information, Mathematical and Computer Modelling, 56, 27-35 (2012).

[8] H. M. Zhang, Some interval-valued 2-tuple linguistic aggregation operators and application in multiattribute group decision making, Applied Mathematical Modelling, 37, 4269-4282 (2013).

[9] Z. S. Xu, An Approach Based on Similarity Measure to Multiple Attribute Decision Making with Trapezoid Fuzzy Linguistic Variables, Fuzzy Systems and Knowledge Discovery, 3613, 110-117 (2005).

[10] K. T. Atanassov, Intuitionistic fuzzy sets, Fuzzy Sets and Systems, 20, 87-96 (1986).

[11] K. T. Atanassov, Intuitionistic fuzzy sets: theory and applications, Heidelberg: Physica-Verlag, (1999).

[12] R. Y. Chen, A problem-solving approach to product design using decision tree induction based on intuitionistic fuzzy, European Journal of Operational Research, 196, 266-272 (2009).

[13] Z. S. Xu, Some similarity measures of intuitionistic fuzzy sets and their applications to multiple attribute decision making, Fuzzy Optimization and Decision Making, 6, 109-121 (2007).

[14] Z. S. Xu, Intuitionistic fuzzy hierarchical clustering algorithms, Journal of Systems Engineering and Electronics, 20, 90-97 (2009).

[15] D. F. Li, Some measures of dissimilarity in intuitionistic fuzzy structures, Journal of Computer and System Sciences, 68, 115-122 (2004).

[16] D. F. Li, Multiattribute decision making models and methods using intuitionistic fuzzy sets, Journal of Computer and System Sciences, 70, 73-85 (2005).

[17] M. M. Xia, Z. S. Xu and B. Zhu, Some issues on intuitionistic fuzzy aggregation operators based on Archimedean t-conorm and t-norm, KnowledgeBased Systems, 31, 78-88 (2012).

[18] J. Q. Wang and J. J. Li, The multi-criteria group decision making method based on multi-granularity intuitionistic two semantics, Science \& Technology Information, 33, 8-9 (2009).

[19] P. D. Liu, Some generalized dependent aggregation operators with intuitionistic linguistic numbers and their application to group decision making, Journal of Computer and System Sciences, 79, 131-143 (2013).

[20] P. D. Liu and F. Jin, Methods for aggregating intuitionistic uncertain linguistic variables and their application to group decision making, Information Sciences, 205, 58-71 (2012).

[21] P. D. Liu, Some geometric aggregation operators based on interval intuitionistic uncertain linguistic variables and their application to group decision making, Applied Mathematical Modelling, 37, 24302444 (2013). 
[22] Y.B. Ju and S.H. Yang, Some aggregation operators with intuitionistic trapezoid fuzzy linguistic numbers and their application to multiple attribute group decision making, Technical Report, (2013).

[23] R. R. Yager, On ordered weighted averaging aggregation operators in multicriteria decision making, IEEE Transactions on Systems, Man and Cybernetics-Part B, 18, 183-190 (1988).

[24] Y. Wang and Z. S. Xu, A new method of giving OWA weights, Mathematics in Practice and Theory, 38, 5161 (2008).

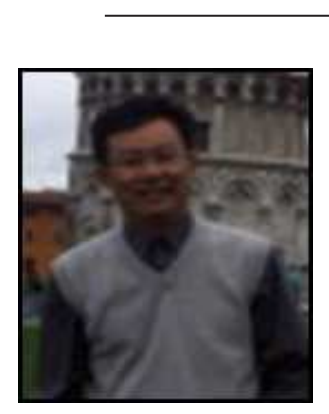

Yanbing Ju is an associate professor of Department of Management Science and Engineering, School of Management and Economics, Beijing Institute of Technology. His research interests are decision making under uncertain environment, system evaluation technology and discrete event system simulation. He received his $\mathrm{PhD}$ degree from Beihang University in 2002.

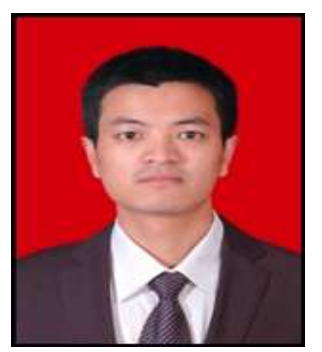

Shanghong Yang

is a $\mathrm{PhD}$ student of Department of Management Science and Engineering, School of Management and Economics, Beijing Institute of Technology. His main research interests are decision making under uncertain environment, strategic management and human resource management

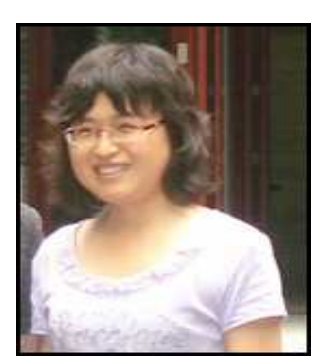

\begin{tabular}{llr}
\multicolumn{1}{c}{ Aihua } & Wang is an \\
Associate & Professor of \\
Department & of Educational \\
Technology, & & Graduate
\end{tabular}

School of Education, Peking

University. Her research interests are information technology in education, education resource sharing and educational decision making. She graduated from Department of Computer Science, Peking University in 2002, receiving $\mathrm{PhD}$ degree. 